\title{
Effects of baicalein on proliferation, apoptosis, migration and invasion of Ewing's sarcoma cells
}

\author{
CONGLIN YE*, XIAOLONG YU*, JIN ZENG, MIN DAI and BIN ZHANG \\ Department of Orthopedics, The First Affiliated Hospital of Nanchang University, Artificial Joints Engineering \\ and Technology Research Center of Jiangxi Province, Nanchang, Jiangxi 330006, P.R. China
}

Received July 30, 2017; Accepted September 25, 2017

DOI: 10.3892/ijo.2017.4148

\begin{abstract}
Ewing's sarcoma (ES) is a rare tumor that is more frequent in pediatric and adolescent age groups. In the past few decades, long-term survival in affected patients has improved due to the success of multimodal therapy. However, longterm survival is inevitably restricted by the late side-effects of chemotherapy. Besides, early metastasis also contributes to the poor prognosis of ES. Recently, traditional Chinese medicines (TCMs) have increasingly attracted interest due to the promising clinical results and fewer side-effects for the treatment of cancers. Among the various TCMs, the root of Scutellaria baicalensis exerts anti-inflammatory properties as a well-known herb in traditional Chinese medicine. Baicalein (5,6,7-trihydroxyflavone) derived from the root of Scutellaria baicalensis is a bioactive compound, which possesses a powerful pro-apoptotic activity in various cancers such as hepatocellular carcinoma and myeloma. However, the effects of baicalein on ES cells remain still unknown. We anticipated that baicalein also has apoptotic activity in ES. The aim of the present study was to investigate the effects of baicalein on viability, apoptosis, migration and invasion of ES cells, and further to elaborate the molecular mechanism of baicalein-induced ES cell apoptosis. We found that baicalein markedly inhibited ES cells viability in a time- and dose-dependent manner, especially SK-ES-1 cells and could promote the apoptosis of ES cells. Additionally, baicalein was capable of upregulating the expression of the pro-apoptotic proteins Bax and cytochrome $c$, reducing the expression of
\end{abstract}

Correspondence to: Professor Min Dai or Professor Bin Zhang, Department of Orthopedics, The First Affiliated Hospital of Nanchang University, Artificial Joints Engineering and Technology Research Center of Jiangxi Province, No. 17 Yong Wai Zheng Street, Nanchang, Jiangxi 330006, P.R. China

E-mail: daimin@medmail.com.cn

E-mail: 13970823907@163.com

${ }^{*}$ Contributed equally

Key words: baicalein, human Ewing's sarcoma, anticancer activity, invasion and migration, apoptosis, mitochondrial pathway the anti-apoptotic protein Bcl-2, elevating the ratio of Bax/ Bcl-2, and triggering the mitochondrial apoptotic pathway, which led to caspase-3 and caspase-9 activation and PARP cleavage. Meanwhile, the activation of caspase- 8 and the death receptor pathway was also observed. Besides, baicalein could reduce ES migration and invasion in vitro, which showed its potential to inhibit ES metastasis, besides contributing to the decrease in the expression of matrix metalloproteinases (MMP)-2 and MMP-9. In conclusion, baicalein has a potent tumor-suppressor activity by inducing cell apoptosis through the mitochondrial apoptotic pathway and the death receptor pathway in ES cells, thus it may serve as a novel and effective candidate agent for ES treatment.

\section{Introduction}

Ewing's sarcoma (ES), characterized by small round cells, is the second most common primary bone and soft tissue malignant tumor that mainly affects children and adolescents (1-3). The initial symptom of the primary ES was usually intermittent pain that was not frequently felt during night. Usually the most important clinical feature was a palpable mass, which was found at the first visit in over one-third of the patients (4). Owing to the development of multiagent systemic chemotherapy and local control methods over the past few decades, overall survival of the patients with localised ES has risen to approximately $75 \%$ (5). However, the multimodality chemotherapy inevitably results in serious toxicity and severe side-effects, such as cardiotoxicity and nephrotoxicity. Furthermore, nearly $25 \%$ of patients with ES have metastatic disease at the time of diagnosis (1). For these patients, cytotoxic chemotherapy has had much less effect on the survival of patients with metastases at diagnosis (5). Furthermore, the effectiveness of chemotherapy is seriously limited by the occurrence of drug resistance (6). Thus, safer and more effective anticancer drugs are urgently needed in clinical practice.

Great efforts have been made to develop new therapeutic agents against cancer using novel bioactive compounds extracted from plants and other natural sources (7-9). Scutellaria baicalensis (SB), known as Huang Qin in China, is widely used to treat various diseases including inflammation, hypertension and bacterial and viral infections with low sideeffects as a traditional Chinese medicine (10). Among over 50 different kinds of flavonoids currently derived and identified 
from the root of SB, baicalein has been shown to exert a potent antitumor and/or pro-apoptotic activity against different types of cancers. Baicalein has been reported to suppress adhesion, migration and invasion of MDA-MB-231 human breast cancer cells (11). Baicalein can also preferentially inhibit HCC tumor growth through inhibition of MEK-ERK signaling and by inducing intrinsic apoptosis (12). Besides, baicalein can lead to suppression of proliferation and induction of apoptosis in human myeloma cells (13). Moreover, baicalein has been demonstrated to suppresses colorectal carcinoma cell invasion via inhibition of the ERK signaling pathways (14). However, there have not yet been any studies on the effects of baicalein on ES.

We therefore assumed that baicalein may also possess an antitumor and/or pro-apoptotic activity in ES. Subsequently, we investigated the effects of baicalein on viability, apoptosis, migration and invasion of human ES SK-ES-1 cells and further expound the related molecular mechanisms.

\section{Materials and methods}

Chemicals. Baicalein was purchased from Sigma-Aldrich (St. Louis, MO, USA). Baicalein was freshly prepared before each experiment and was solubilized with dimethyl sulfoxide (DMSO). The final concentration of DMSO in the medium was $<0.1 \%(\mathrm{v} / \mathrm{v})$ in the treatment range $(20-40 \mu \mathrm{M})$ and showed no influence on cell growth (data not shown).

Reagents. RPMI-1640 medium, phosphate-buffered saline (PBS), DMSO, bovine serum albumin (BSA) and Cell Counting kit-8 (CCK-8) were purchased from TransGen Biotech, Inc. (Beijing, China). Fetal bovine serum (FBS) was purchased from HyClone Laboratoriess (Thermo Fisher Scientific, Waltham, MA, USA). An Annexin V-FITC/PI (propidium iodide) Apoptosis Detection kit and Matrigel were purchased from Becton-Dickinson (San Jose, CA, USA). The Transwell invasion chambers were purchased from Costar (Cambridge, MA, USA). Crystal violet staining solution and methanol were purchased from Beyotime Institute of Biotechnology (Haimen, China). Hoechst 33258 staining kit was purchased from Keygen Nanjing KeyGen Biotech Co., Ltd. (Nanjing, China). Antibodies against Bax, Bcl-2, cytochrome $c$, caspase-3, procaspase-9, PARP and $\beta$-actin were purchased from Abcam (Cambridge, UK). Antibodies against cleaved caspase-8, MMP-2 and MMP-9 were purchased from Wuhan Boster Biological Technology, Ltd. (Wuhan, China). Horseradish peroxidase (HRP)-conjugated secondary antibodies were purchased from TransGen Biotech.

Cell culture. Human ES cell lines, SK-ES-1 and RD-ES, were obtained from the American Type Culture Collection (ATCC; Manassas, VA, USA). Cells were cultured in RPMI-1640 medium supplemented with $10 \%$ (v/v) FBS, $100 \mathrm{U} / \mathrm{ml}$ penicillin and $100 \mu \mathrm{g} / \mathrm{ml}$ streptomycin. The cells were maintained in a humidified atmosphere containing $5 \% \mathrm{CO}_{2}$ at $37^{\circ} \mathrm{C}$. The cells used in the present study were subjected to $<20$ passages, and all cells used in this study were in the logarithmic phase.

Cell viability by $C C K-8$ assay. Cell viability was determined using CCK-8 assay. The cells were cultured in 96-well plates $\left(5 \times 10^{3}\right.$ cells/well). The cells were treated with baicalein at different final concentrations $(5,10,20,40,80$ and $160 \mu \mathrm{M})$ for 24,36 and $48 \mathrm{~h}$, respectively, and the control cells were treated with DMSO $<0.1 \%(\mathrm{v} / \mathrm{v})$. After indicated cultivation time, the medium was changed to normal culture medium containing $10 \% \mathrm{CCK}-8$ solution at $37^{\circ} \mathrm{C}$ for $1 \mathrm{~h}$. Subsequently, the absorbance was measured at $450 \mathrm{~nm}$ using a Universal microplate reader (EL800; Bio-Tek Instruments, Inc., Winooski, VT, USA). Cell viability as percent viability was calculated by comparing the absorbance of treated cells vs. the untreated ones.

Hoechst 33258 staining of SK-ES-1 cells. Cells were incubated with 0,20 and $40 \mu \mathrm{M}$ of baicalein in 6-well plates for $24 \mathrm{~h}$. Then cells were fixed with $4 \%$ paraformaldehyde for $30 \mathrm{~min}$ at $25^{\circ} \mathrm{C}$. Later on, the cells were washed three times with ice-cold phosphate-buffered saline (PBS) and stained with $10 \mathrm{mg} / 1$ Hoechst 33258 solution for $10 \mathrm{~min}$ in the dark at $25^{\circ} \mathrm{C}$. Subsequently, the stained nuclei were observed under a fluorescence microscope (Olympus Corp., Tokyo, Japan) with excitation at $350 \mathrm{~nm}$ and emission at $460 \mathrm{~nm}$ (original magnification, $\mathrm{x} 200$ ).

Analysis of cell apoptosis by Annexin V-FITC/PI staining assay. Flow cytometry was conducted to assess the apoptosis induced by baicalein. SK-ES-1 cells incubated with 0,20 and $40 \mu \mathrm{M}$ of baicalein for $24 \mathrm{~h}$ were collected, washed twice with ice-cold PBS, and resuspended in $1 \mathrm{X}$ binding buffer at a concentration of $1 \times 10^{6}$ cells $/ \mathrm{ml}$. The cell suspension $(100 \mu \mathrm{l})$ was incubated with $1 \mu \mathrm{l}$ of Annexin V-FITC and $2 \mu \mathrm{l}$ of a PI solution in the dark for $15 \mathrm{~min}$ at $25^{\circ} \mathrm{C}$. The samples were analyzed on a FACSVerse flow cytometer (BD Biosciences, San Jose, CA, USA) after the addition of $150 \mu \mathrm{l}$ of $1 \mathrm{X}$ binding buffer. The apoptosis rates were analyzed using the FlowJo 7.6 software (Tree Star, Inc., Ashland, OR, USA).

Western blot analysis. SK-ES-1 cells were cultured in 6 -well plates at a concentration of $3 \times 10^{5}$ cells/well. After treatment with 0,20 and $40 \mu \mathrm{M}$ of baicalein for $24 \mathrm{~h}$, the cells were collected and lysed in RIPA buffer containing protease inhibitor cocktail (Sigma-Aldrich). Subsequently the samples were centrifuged at $17,105.6 \mathrm{x} \mathrm{g}$ for $10 \mathrm{~min}$ at $4^{\circ} \mathrm{C}$ to remove cell debris using a Universal $320 \mathrm{R}$ centrifuge (Hettich Corp., Germany). Then the supernatants were collected, and the protein concentrations were determined by a BCA protein assay kit (Thermo Fisher Scientific). Identical quantities of proteins were loaded, separated by sodium dodecyl sulfate polyacrylamide gel electrophoresis, and then transferred onto polyvinylidene difluoride membranes. The membranes were incubated with $5 \%$ skim milk for $2 \mathrm{~h}$. Then the membranes were incubated overnight at $4^{\circ} \mathrm{C}$ with the primary antibodies. Subsequently, the membranes were washed three times for 10 min with $1 \mathrm{X}$ TBST buffer and incubated for $2 \mathrm{~h}$ with horseradish peroxidase-conjugated secondary antibodies at $25^{\circ} \mathrm{C}$ for $2 \mathrm{~h}$. Finally, antigenantibody complexes were detected using an enhanced chemiluminescence detection system (Amersham Life Science, Inc., Pittsburg, PA, USA). The gray values of the bands were analyzed using the ImageJ software (National Institutes of Health, Bethesda, MD, USA). 

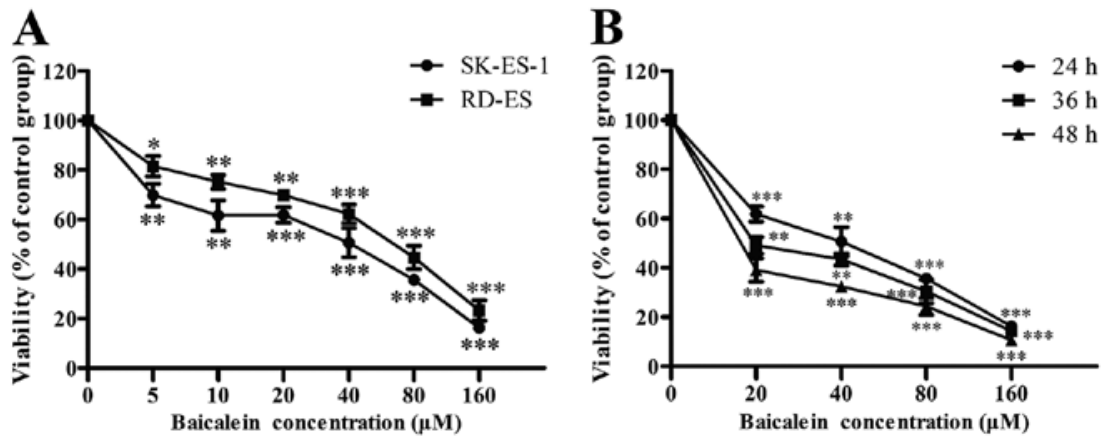

Figure 1. Analysis of cell viability. (A) Effect of baicalein on the viability of SK-ES-1 and RD-ES cells. SK-ES-1 and RD-ES cells were treated with baicalein at different concentrations for $24 \mathrm{~h}$. (B) Baicalein suppressed the viability of SK-ES-1 in a time- and dose-dependent manner. SK-ES-1 cells were treated with baicalein at different concentrations for 24,36 and $48 \mathrm{~h}$. The data are presented as the means \pm SD of three independent experiments. Significant differences are indicated by ${ }^{*} \mathrm{P}<0.05 ;{ }^{* *} \mathrm{P}<0.01 ;{ }^{* * *} \mathrm{P}<0.001$.
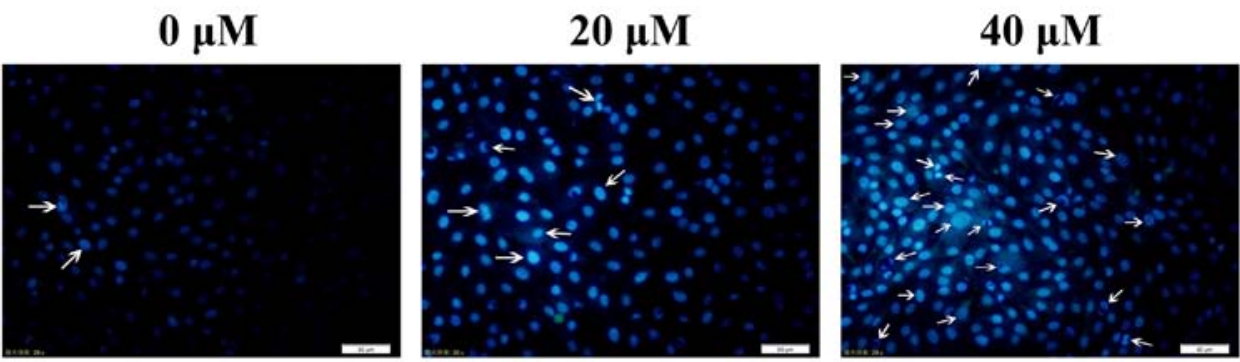

Figure 2. Hoechst 33258 staining of SK-ES-1 cells. Apoptotic nuclei manifested nuclear chromosomal condensation and fragmentation brightly stained by Hoechst 33258 (24 h). The arrows indicated the nuclear changes in cells (original magnification, x200).

Cell migration assay. Migration of SK-ES-1 cells was measured using wound healing assays. SK-ES-1 cells were seeded in 6 -well culture plates $\left(3 \times 10^{5}\right.$ cells/well $)$ to form a confluent monolayer, and then cells were wounded with a sterile $100-\mu 1$ pipette tip. The cells in the plates were treated with baicalein at final concentrations of 0,20 and $40 \mu \mathrm{M}$, and then incubated in fresh RPMI-1640 medium without FBS for $24 \mathrm{~h}$. Scratch wounds were then inspected using a phasecontrast microscope (Olympus) and images of each wound were taken (original magnification, $\mathrm{x} 40$ ).

Cell invasion assay. Invasion of SK-ES-1 cells was examined using Matrigel-coated Transwell cell culture chambers $(8 \mu \mathrm{m}$ pore size). Briefly, the membranes in each chamber were coated with $100 \mu \mathrm{g} / \mathrm{ml}$ Matrigel, after which $5 \times 10^{4}$ cells were seeded into the upper chamber and treated with baicalein $(0$, 20 and $40 \mu \mathrm{M})$, and the lower wells were filled with RPMI1640 medium supplemented with $20 \%$ (v/v) FBS in 24-well culture plates. All the cells were incubated for $24 \mathrm{~h}$ at $37^{\circ} \mathrm{C}$ in an incubator containing $5 \% \mathrm{CO}_{2}$. Subsequently, the noninvaded cells in the upper chamber were gently removed with a cotton swab, whereas the cells attached to the lower surface were fixed with precooled methanol and stained with $0.1 \%$ crystal violet solution. Five fields of each chamber were randomly selected, and the cell numbers were counted under a microscope (original magnification, x100). The numbers of invaded cells were analyzed by the ImageJ software.

Statistical analysis. Data are expressed as the mean \pm standard deviation (SD) of three independent experiments. Statistical analysis was performed using the SPSS 19.0 software (SPSS, Inc., Chicago, IL, USA). Student's t-test (two-tailed) was used to analyze the differences between the two groups. $\mathrm{P}<0.05$ was considered to be statistically significant.

\section{Results}

Baicalein inhibits the viability of ES cells. To study the effects of baicalein on the viability of ES cells, SK-ES-1 and RD-ES cells were exposed to different concentrations of baicalein for $24 \mathrm{~h}$, and their viability was determined by CCK-8 assay. As shown in Fig. 1A, baicalein significantly repressed the viability of SK-ES-1 and RD-ES cells in a dose-dependent manner $(\mathrm{P}<0.05)$. Besides, SK-ES-1 cells were more sensitive to baicalein. The $\mathrm{IC}_{50}$ value for the SK-ES-1 cells treated with baicalein was $28.1 \mu \mathrm{M}$ at $24 \mathrm{~h}$. Furthermore, it was observed that baicalein suppressed the viability of SK-ES-1 in a timeand dose-dependent manner $(\mathrm{P}<0.01)$ (Fig. 1B). Subsequently, SK-ES-1 cells were treated with baicalein at the concentrations of 0,20 and $40 \mu \mathrm{M}$ for $24 \mathrm{~h}$ in the following assays.

Baicalein causes the nuclear changes of SK-ES-1 cells. SK-ES-1 cells were treated with baicalein $(0,20$ and $40 \mu \mathrm{M})$ for $24 \mathrm{~h}$. It was found that baicalein produced nuclear chromosomal condensation and fragmentation, which are the typical morphological features of apoptotic cells, in SK-ES-1 cells stained with Hoechst 33258 in a dose-dependent manner. These findings suggested that cell death occurred through apoptosis (Fig. 2). The arrows in Fig. 2 indicated the nuclear changes in cells. Baicalein induces apoptosis in SK-ES-1 cells. 

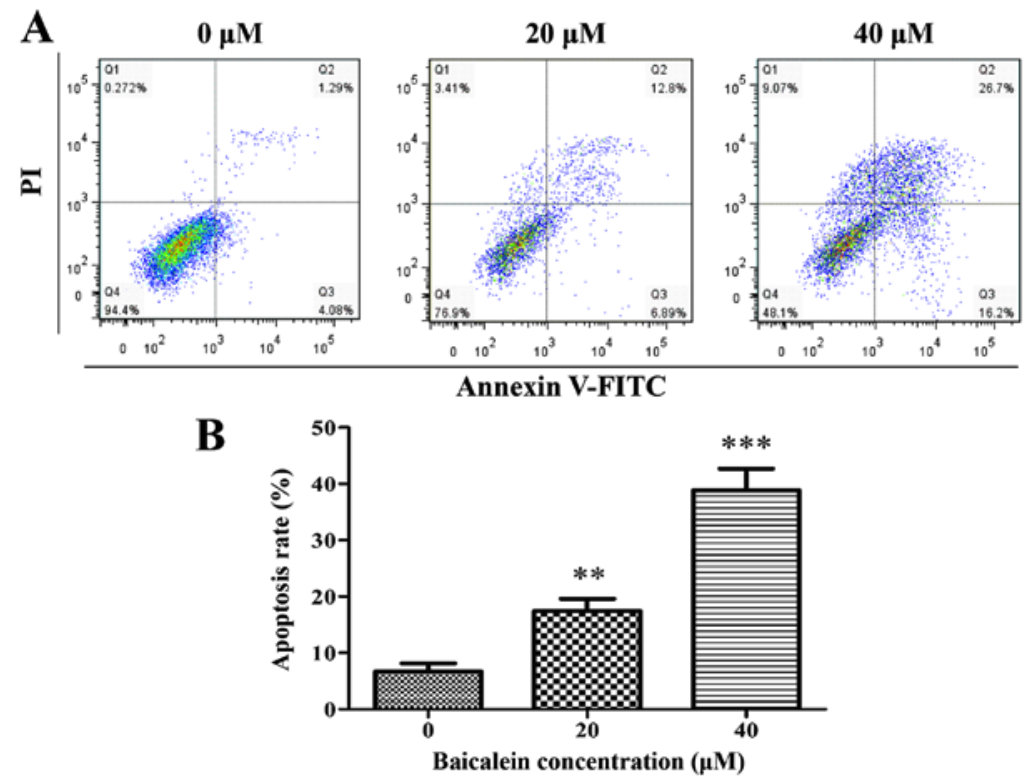

Figure 3. Annexin V-FITC/PI staining assay. (A) Representative graphs obtained by flow cytometric analysis after double-staining with Annexin V-FITC/PI. (B) The apoptosis rate in the control group and SK-ES-1 cells treatment with baicalein $(24 \mathrm{~h})$. Significant differences are indicated by ${ }^{* * *} \mathrm{P}<0.01{ }^{*{ }^{* * * *}} \mathrm{P}<0.001$.

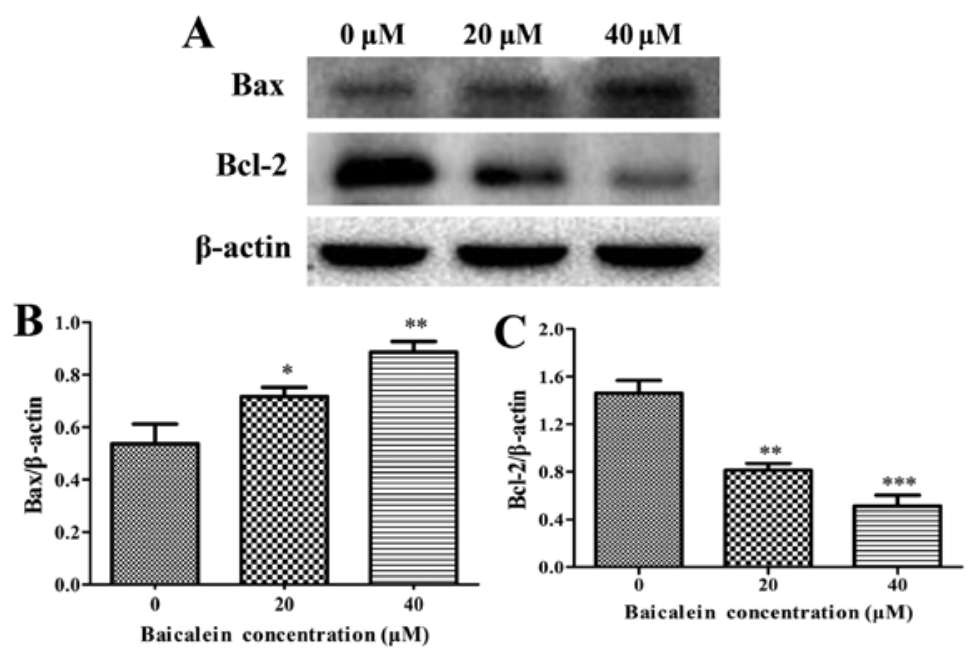

Figure 4. Effect of baicalein on the protein expression levels of Bax and Bcl-2 in SK-ES-1 cells. (A) The protein expression levels of Bax and Bcl-2 by western blot analysis. (B and C) Quantification of Bax and Bcl-2 using the ImageJ software. The data shown are the means \pm SD, and significant differences are indicated by ${ }^{*} \mathrm{P}<0.05,{ }^{* *} \mathrm{P}<0.01,{ }^{* * *} \mathrm{P}<0.001$.

Cell apoptosis was measured by flow cytometry by double labeling with Annexin V-FITC/PI. Representative graphs, which were obtained by flow cytometric analysis of SK-ES-1 cells treated with baicalein at 0,20 and $40 \mu \mathrm{M}$ for $24 \mathrm{~h}$, are shown in Fig. 3A. The apoptosis rate (the sum of apoptotic rates of both early stage and late stage) in the control group and SK-ES-1 cells treatment with baicalein at 20 and $40 \mu \mathrm{M}$ for $24 \mathrm{~h}$, was $6.7 \pm 1.5,17.4 \pm 2.2$ and $38.9 \pm 3.8 \%$, respectively. Compared with the control group, the apoptosis rate in the treatment group significantly increased $(\mathrm{P}<0.01)$. The treatment group was dose-dependent (Fig. 3B).

Effects of baicalein on the expression of cell apoptosis-related proteins. The expression levels of anti-apoptotic Bcl-2, proapoptotic Bax, cytochrome $c$, caspase- 3 , caspase- 8 , caspase- 9 , and PARP were assessed by western blot analysis to determine the molecular mechanism on baicalein induced apoptosis of SK-ES-1 cells. The results showed that baicalein treatment caused a remarkable increase in the expression of Bax and the release of cytochrome $c$, whereas a decrease in Bcl-2 expression when compared to in the control group $(\mathrm{P}<0.05)$ (Figs. 4 and $5 \mathrm{~A}$ and $\mathrm{B}$ ). Besides, the expression level of procaspase- 9 was downregulated, whereas the cleaved caspase- 8 and the cleaved caspase- 3 markedly increased in a dose-dependent manner $(\mathrm{P}<0.05)$ (Figs. 5 and 6). The cleavage of PARP, a key substrate of activated caspase-3, remarkably increased in a dose-dependent manner (Fig. 6). These findings revealed that baicalein induced ES cell apoptosis by activation of caspase-3, caspase- 8 and caspase- 9 .

Baicalein inhibits cell migration and invasion in SK-ES-1 cells. The effects of baicalein on the migration and invasion 

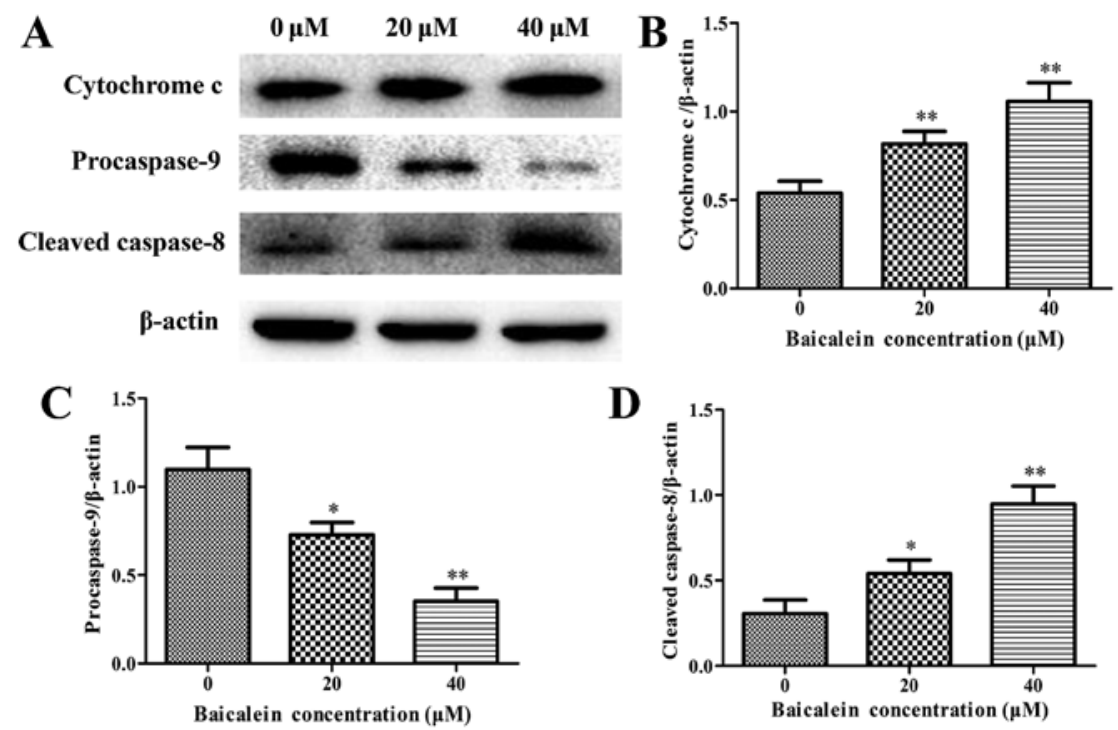

Figure 5. Effect of baicalein on the protein expression levels of cytochrome $c$, procaspase- 9 and cleaved caspase-8 in SK-ES-1 cells was analyzed by western blot analysis. (A) The expression levels of cytochrome $c$, procaspase- 9 and cleaved caspase- 8 by the western blot analysis. (B-D) Quantification of cytochrome $c$, procaspase- 9 and cleaved caspase- 8 using the ImageJ software. The data shown are the means \pm SD and significant differences are indicated by ${ }^{*} \mathrm{P}<0.05,{ }^{* *} \mathrm{P}<0.01$.

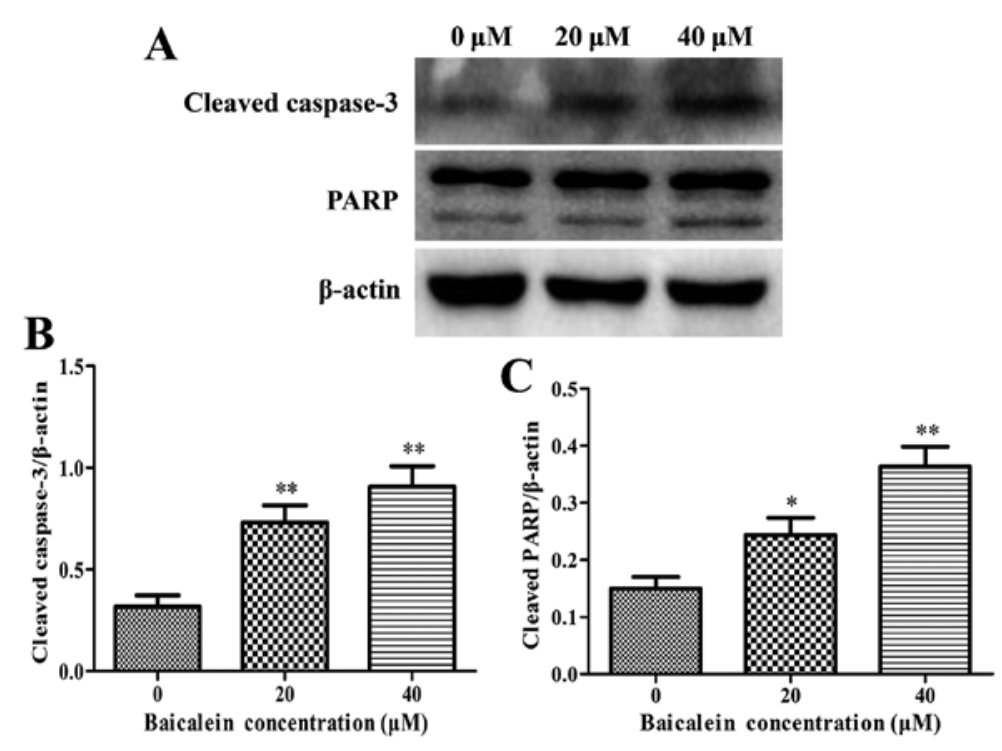

Figure 6. Effect of baicalein on the protein expression levels of cleaved caspase-3 and PARP in SK-ES-1 cells. (A) The expression levels of cleaved caspase-3 and PARP by the western blot analysis. (B-C) Quantification of cleaved caspase-3 and PARP by the ImageJ software. The data shown are the means \pm SD, and significant differences are indicated by ${ }^{*} \mathrm{P}<0.05,{ }^{* *} \mathrm{P}<0.01$.

of SK-ES-1 cells were detected by wound healing assays and Boyden chamber Transwell assays, respectively. As shown in Fig. 7A, baicalein significantly repressed the migration and invasion of SK-ES-1 cells in a dose-dependent manner $(\mathrm{P}<0.01)$.

Baicalein decreases the expression levels of MMP-2 and $M M P$-9. It is well-known that matrix metalloproteinases (MMPs), among which MMP-2 and MMP-9 play the leading roles, can facilitate the invasion and migration of tumor cells. It was found that baicalein treatment group caused a significant decrease in MMP-2 and MMP-9 when compared with the control group $(\mathrm{P}<0.01)$ (Fig. 8). These results indicated that baicalein inhibited migration and invasion in SK-ES-1 cells through downregulating the expression of MMP-2 and MMP-9.

\section{Discussion}

Apoptosis (a programmed cell death), is an innate process to eliminate abnormal or redundant cells in mammals and is considered to be an important mechanism in the action of many anticancer drugs (15). Growing evidence shows that various kinds of herbal medicines and compounds derived from natural products with antitumor effects can induce apoptosis in various tumor cells (15-17). Accumulating evidence demonstrates that baicalein can induce apoptosis in a wide variety of cancer cell lines. Baicalein can induce apoptosis of the human 

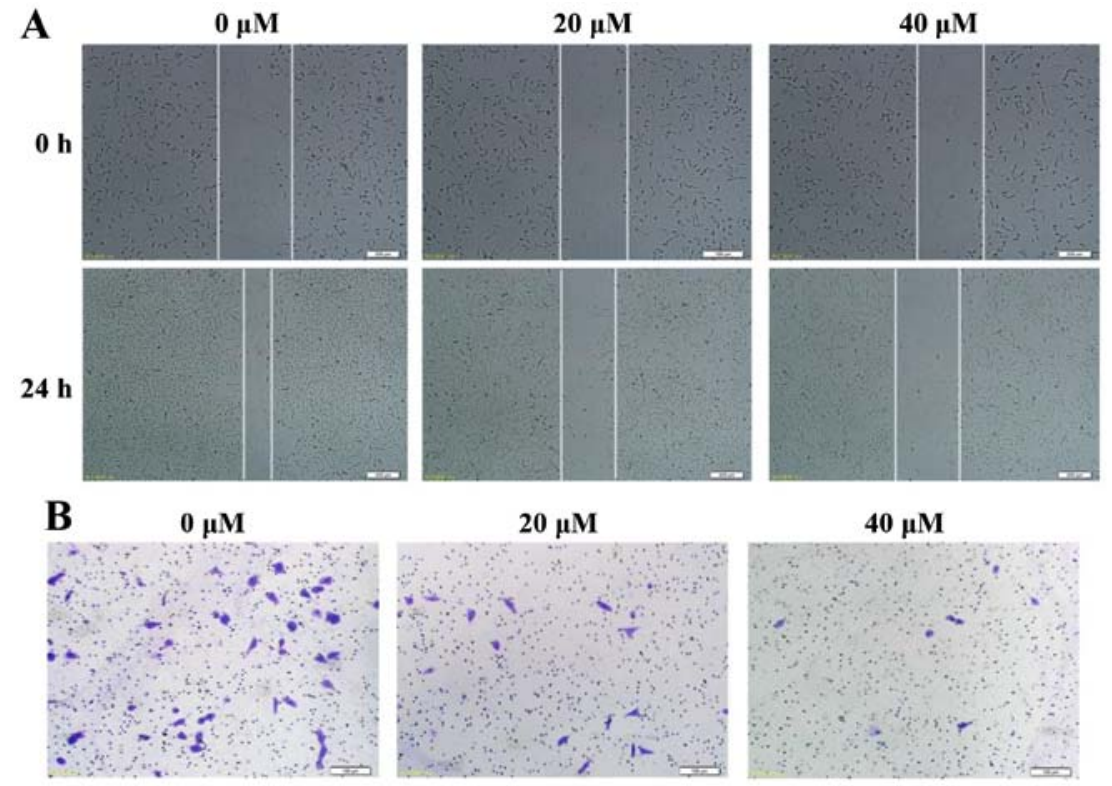

$20 \mu \mathrm{M}$

$40 \mu \mathrm{M}$
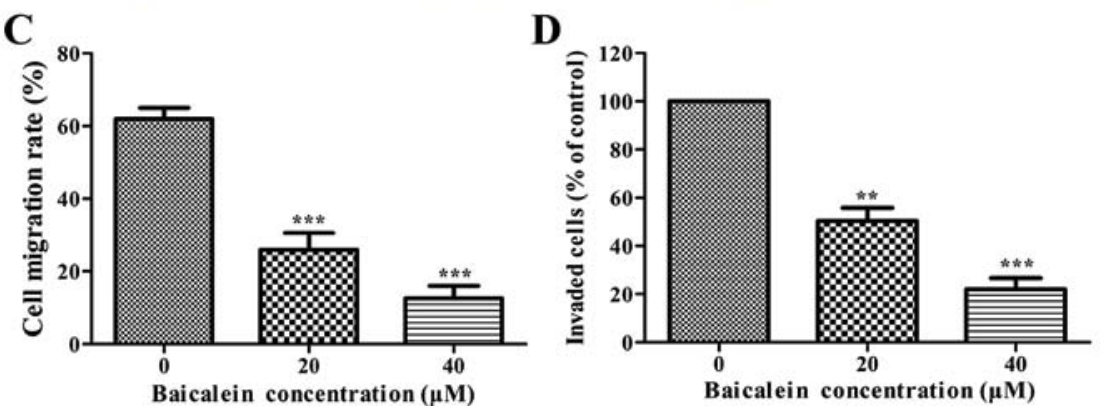

Figure 7. Effect of baicalein on cell migration and invasion in SK-ES-1 cells (24 h). (A) Cell migration was determined by wound healing assays (original magnification, x40). (B) Cell invasion was examined using Matrigel-coated Transwell cell culture chambers (original magnification, x100). (C) Quantification of the migration ability in SK-ES-1 cells by the ImageJ software. (D) Quantification of the invaded cells in SK-ES-1 cells by the ImageJ software. The data shown was the means $\pm \mathrm{SD}$ and significant differences were indicated by ${ }^{* *} \mathrm{P}<0.01,{ }^{* * *} \mathrm{P}<0.001$.

A
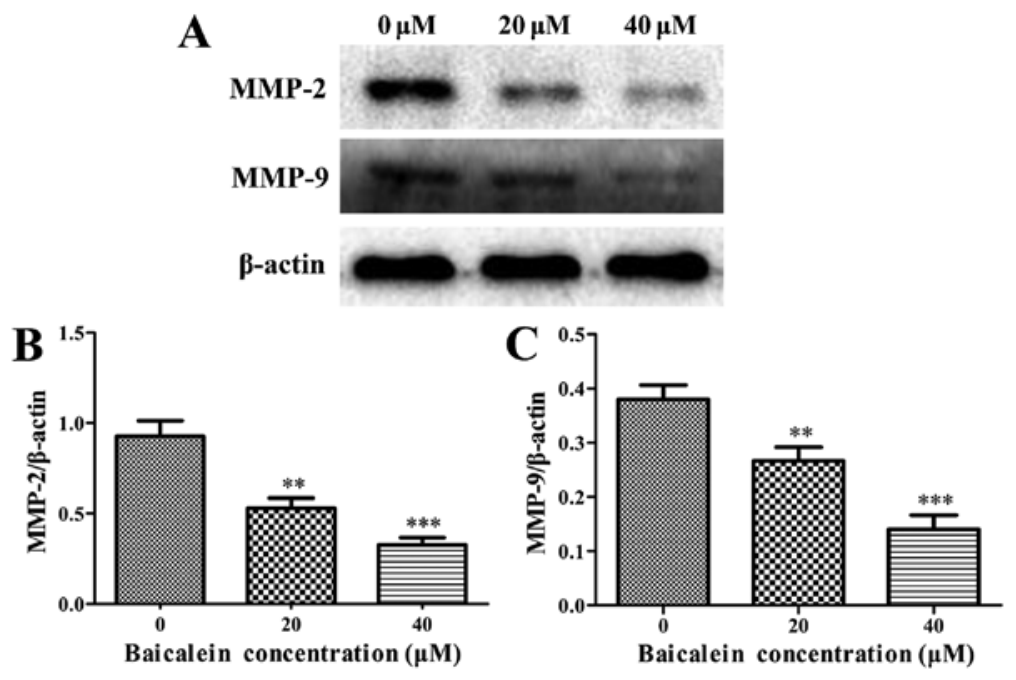

Figure 8. Effect of baicalein on the protein expression levels of MMP-2 and MMP-9 in SK-ES-1 cells. (A) The protein expression levels of MMP-2 and MMP-9 by western blot analysis. (B and C) Quantification of MMP-2 and MMP-9 using the ImageJ software. The data shown are the means \pm SD and significant differences are indicated by ${ }^{* *} \mathrm{P}<0.01,{ }^{* * * *} \mathrm{P}<0.001$.

hepatoblastoma G2 cell line by mitochondrial dysfunction and Bcl-2 regulation (18). Besides, as a lipoxygenase inhibitor, baicalein can block both the 5-LOX and 12-LOX pathways and therefore induce apoptosis in breast cancer cells through the cytochrome $c$ release and caspase- 9 activation, with changes in the levels of Bcl-2 family proteins (19). Furthermore, it has 
been reported that baicalein is an effective anti-HCC agent with low cytotoxicity to normal liver cells (12). Given the above, baicalein is likely to exert potent antitumor effects with few side-effects. Hence, we tried to explore the exact effects of baicalein on ES and the related molecular mechanisms, which have remained obscure.

It was seen from the present study that baicalein significantly inhibited SK-ES-1 cell viability of human ES and induced apoptosis in SK-ES-1 cells in a time- and dose-dependent manner. Besides, baicalein can suppress the migration and invasion of SK-ES-1 cells in a dose-dependent manner. All these findings are consistent with the reported literature in other types of tumors, which consider baicalein as an anticancer substance. To the best of our knowledge, this is the first study to explore the effects of baicalein on ES in vitro.

Then, we further explored the possible mechanisms of apoptosis, which is the most important process in the function of many anticancer drugs. Apoptosis occurs through two different pathways: the intrinsic pathway and extrinsic pathway, which was regulated by caspase- 9 and caspase- 8 , respectively $(20,21)$. As a pivotal process in apoptosis, caspase activation is critical for both extrinsic and intrinsic pathways. In the intrinsic pathway (also known as the mitochondrial pathway), the activation of downstream caspases is regulated by members of the Bcl-2 family. Pro-apoptotic Bax-like proteins in the regulation of the formation of pores in the mitochondria, which results in the release of cytochrome $c$. Anti-apoptotic Bcl-2-like proteins exert the completely opposite effect. Following the increase of the ratio of $\mathrm{Bax} / \mathrm{Bcl}-2$, many apoptogenic proteins are released from the mitochondrial intermembrane space, such as cytochrome $c$ that can further activate caspase-9. Caspase-3, an important executioner caspase, is activated after the activation of caspase- 8 and caspase- 9 . Subsequently, active caspase- 3 leads to the cleavage or degradation of some key cellular substrates including PARP, which results in the subsequent apoptosis (22-28). The extrinsic pathway (also known as the death receptor pathway), functioning in other ways, activates the death receptor (known as Fas/FasL) on cell surface and then induces caspase-8 activation (29-31). Our results indicated that baicalein-induced apoptosis was accompanied with the increase of the release of cytochrome $c, \mathrm{Bax} / \mathrm{Bcl}-2$ ratio, and the activation of caspase-3, caspase- 8 and caspase- 9 . Moreover, the cleavage of PARP significantly increased in a dose-dependent manner. The present data suggests that baicalein induces apoptosis in ES cells through the mitochondrial apoptotic (intrinsic) pathway and the death receptor (extrinsic) pathway.

Nearly $25 \%$ of patients with ES have metastatic disease at the time of diagnosis. Besides, early metastasis also contributes to the poor prognosis of ES. Therefore, we decided to test also migration and invasion after apoptosis. Besides, the relationship between the inhibition of migration/invasion and increased apoptosis could be negatively correlated as we speculated. It was found that baicalein inhibited the migration and invasion of SK-ES-1 cells in a dose-dependent manner. Western blot assays showed a marked reduction in the expression levels of MMP-2 and MMP-9, which are closely related with tumor invasion and metastasis (32-34). Hence, it was demonstrated that baicalein inhibits ES cell migration and invasion by decreasing MMP-2 and MMP-9 expression.
There are some limitations to the present study. First, the study included only in vitro experiments. To further clarify the effects of baicalein on ES, studies on the in vivo effects of baicalein on SK-ES-1 xenograft tumors in nude mice are necessary. To finally assess the involvement of caspase in cell death, experiments in the presence of caspase inhibitor Z-VAD-FMK should be performed. Since this study focused on merely SK-ES-1 cell line, we need to conduct the experiments on other ES cell lines. It has been shown that baicalein can inhibit tumors by targeting different proteins or signal pathways, such as c-MYC, Wnt signaling pathway and TGF- $\beta$ signaling pathway $(35,36)$. However, we did not try to discover a target of baicalein in ES.

In summary, baicalein inhibits ES cell viability and induces apoptosis through both the mitochondrial apoptotic pathway and the death receptor pathway. Furthermore, baicalein can suppress ES cell migration and invasion by decreasing MMP-2 and MMP-9 expression. Taken together, the conclusion from this study provides in vitro evidence to support baicalein as an efficient candidate agent for the chemoprevention and/or treatment of ES progression. In addition, studies on the in vivo effect of baicalein on SK-ES-1 xenograft tumors in nude mice are in progress.

\section{Acknowledgements}

The present study was supported by the Natural Science Foundation of Jiangxi Province (20171BAB205059), the Foundation of the Health Department of Jiangxi Province on Traditional Chinese Medicine (2016A073) and the Innovation Fund Designated for Graduate Students of Jiangxi Province (YC2016-S056).

\section{References}

1. Esiashvili N, Goodman M and Marcus RB Jr: Changes in incidence and survival of Ewing sarcoma patients over the past 3 decades: Surveillance Epidemiology and End Results data. J Pediatr Hematol Oncol 30: 425-430, 2008.

2. Gaspar N, Hawkins DS, Dirksen U, Lewis IJ, Ferrari S, Le Deley MC, Kovar H, Grimer R, Whelan J, Claude L, et al: Ewing sarcoma: Current management and future approaches through collaboration. J Clin Oncol 33: 3036-3046, 2015.

3. Iwamoto Y: Diagnosis and treatment of Ewing's sarcoma. Jpn J Clin Oncol 37: 79-89, 2007.

4. Widhe B and Widhe T: Initial symptoms and clinical features in osteosarcoma and Ewing sarcoma. J Bone Joint Surg Am 82: 667-674, 2000.

5. Balamuth NJ and Womer RB: Ewing's sarcoma. Lancet Oncol 11: 184-192, 2010.

6. Newman DJ, Cragg GM, Gao Y, Du Z, Wang Y, Cheng P, Chen A and Huang H: Natural products as sources of new drugs over the last 25 years. J Nat Prod 70: 461-477, 2007.

8. Cassileth B, Yeung KS and Gubili J: Herbs and other botanicals in cancer patient care. Curr Treat Options Oncol 9: 109-116, 2008.

9. Yu X, Zhou X, Fu C, Wang Q, Nie T, Zou F, Guo R, Liu H, Zhang B and Dai M: Celastrol induces apoptosis of human osteosarcoma cells via the mitochondrial apoptotic pathway. Oncol Rep 34: 1129-1136, 2015.

10. Li-Weber M: New therapeutic aspects of flavones: The anticancer properties of Scutellaria and its main active constituents Wogonin, Baicalein and Baicalin. Cancer Treat Rev 35: 57-68, 2009.

11. Wang L, Ling Y, Chen Y, Li CL, Feng F, You QD, Lu N and Guo QL: Flavonoid baicalein suppresses adhesion, migration and invasion of MDA-MB-231 human breast cancer cells. Cancer Lett 297: 42-48, 2010. 
12. Liang RR, Zhang S, Qi JA, Wang ZD, Li J, Liu PJ, Huang C, Le XF, Yang J and Li ZF: Preferential inhibition of hepatocellular carcinoma by the flavonoid Baicalein through blocking MEK-ERK signaling. Int J Oncol 41: 969-978, 2012.

13. Ma Z, Otsuyama K, Liu S, Abroun S, Ishikawa H, Tsuyama N, Obata M, Li FJ, Zheng X, Maki Y, et al: Baicalein, a component of Scutellaria radix from Huang-Lian-Jie-Du-Tang (HLJDT), leads to suppression of proliferation and induction of apoptosis in human myeloma cells. Blood 105: 3312-3318, 2005.

14. Chai Y, Xu J and Yan B: The anti-metastatic effect of baicalein on colorectal cancer. Oncol Rep 37: 2317-2323, 2017.

15. Zhang K, Wang X, Wang C, Zheng H, Li T, Xiao S, Wang M, Fei C, Zhang L and Xue F: Investigation of quinocetone-induced mitochondrial damage and apoptosis in HepG2 cells and compared with its metabolites. Environ Toxicol Pharmacol 39: 555-567, 2015.

16. Zhang J, Song J, Wu D, Wang J and Dong W: Hesperetin induces the apoptosis of hepatocellular carcinoma cells via mitochondrial pathway mediated by the increased intracellular reactive oxygen species, ATP and calcium. Med Oncol 32: 101, 2015.

17. Pieme CA, Santosh GK, Tekwu EM, Askun T, Aydeniz H, Ngogang JY, Bhushan S and Saxena AK: Fruits and barks extracts of Zanthozyllum heitzii a spice from Cameroon induce mitochondrial dependent apoptosis and Go/G1 phase arrest in human leukemia HL-60 cells. Biol Res 47: 54, 2014.

18. Chang WH, Chen $\mathrm{CH}$, Gau RJ, Lin CC, Tsai CL, Tsai K and Lu FJ: Effect of baicalein on apoptosis of the human Hep G2 cell line was induced by mitochondrial dysfunction. Planta Med 68: 302-306, 2002

19. Tong WG, Ding XZ and Adrian TE: The mechanisms of lipoxygenase inhibitor-induced apoptosis in human breast cancer cells. Biochem Biophys Res Commun 296: 942-948, 2002.

20. Hengartner MO: The biochemistry of apoptosis. Nature 407: 770-776, 2000.

21. Lorenzo HK and Susin SA: Therapeutic potential of AIF-mediated caspase-independent programmed cell death. Drug Resist Updat 10: 235-255, 2007.

22. Chang HY and Yang X: Proteases for cell suicide: Functions and regulation of caspases. Microbiol Mol Biol Rev 64: 821-846, 2000.

23. Cao X, Bennett RL and May WS: c-Myc and caspase- 2 are involved in activating Bax during cytotoxic drug-induced apoptosis. J Biol Chem 283: 14490-14496, 2008.
24. Stennicke HR and Salvesen GS: Properties of the caspases. Biochim Biophys Acta 1387: 17-31, 1998.

25. Hui KK, Kanungo AK, Elia AJ and Henderson JT: Caspase-3 deficiency reveals a physiologic role for Smac/DIABLO in regulating programmed cell death. Cell Death Differ 18: 1780-1790, 2011 .

26. Gillies LA and Kuwana T: Apoptosis regulation at the mitochondrial outer membrane. J Cell Biochem 115: 632-640, 2014.

27. Jourdain A and Martinou JC: Mitochondrial outer-membrane permeabilization and remodelling in apoptosis. Int J Biochem Cell Biol 41: 1884-1889, 2009.

28. Wood WG, Igbavboa U, Muller WE and Eckert GP: Statins, Bcl-2, and apoptosis: Cell death or cell protection? Mol Neurobiol 48: 308-314, 2013.

29. Fulda S and Debatin KM: Extrinsic versus intrinsic apoptosis pathways in anticancer chemotherapy. Oncogene 25: 4798-4811, 2006.

30. Villa-Morales M and Fernández-Piqueras J: Targeting the Fas/ FasL signaling pathway in cancer therapy. Expert Opin Ther Targets 16: 85-101, 2012.

31. Gordon $\mathrm{N}$ and Kleinerman ES: Aerosol therapy for the treatment of osteosarcoma lung metastases: Targeting the Fas/FasL pathway and rationale for the use of gemcitabine. J Aerosol Med Pulm Drug Deliv 23: 189-196, 2010.

32. Li H, Zhang K, Liu LH, Ouyang Y, Bu J, Guo HB and Xiao T: A systematic review of matrix metalloproteinase 9 as a biomarker of survival in patients with osteosarcoma. Tumour Biol 35: 5487-5491, 2014.

33. Wang J, Shi Q, Yuan TX, Song QL, Zhang Y, Wei Q, Zhou L, Luo J, Zuo G, Tang M, et al: Matrix metalloproteinase 9 (MMP-9) in osteosarcoma: Review and meta-analysis. Clin Chim Acta 433: 225-231, 2014.

34. Shang HS, Chang JB, Lin JH, Lin JP, Hsu SC, Liu CM, Liu JY, Wu PP, Lu HF, Au MK, et al: Deguelin inhibits the migration and invasion of U-2 OS human osteosarcoma cells via the inhibition of matrix metalloproteinase-2/-9 in vitro. Molecules 19: 16588-16608, 2014.

35. He N and Zhang Z: Baicalein suppresses the viability of MG-63 osteosarcoma cells through inhibiting c-MYC expression via Wnt signaling pathway. Mol Cell Biochem 405: 187-196, 2015.

36. Chen F, Zhuang M, Peng J, Wang X, Huang T, Li S, Lin M, Lin H, $\mathrm{Xu} \mathrm{Y,} \mathrm{Li} \mathrm{J,} \mathrm{et} \mathrm{al:} \mathrm{Baicalein} \mathrm{inhibits} \mathrm{migration} \mathrm{and} \mathrm{invasion} \mathrm{of}$ gastric cancer cells through suppression of the TGF- $\beta$ signaling pathway. Mol Med Rep 10: 1999-2003, 2014. 